\title{
Assessment of the Bacteriological Profile and Antibiotic Susceptibility Patterns of Wastewater in Health Facilities of Ethiopia
}

\author{
Belayneh Regasa Dadi (D, Eyayu Girma, Mheret Tesfaye, and Mohamed Seid \\ Department of Medical Microbiology, Arba Minch University, Arba Minch, Ethiopia \\ Correspondence should be addressed to Belayneh Regasa Dadi; belayjanimen@gmail.com
}

Received 13 March 2021; Revised 8 June 2021; Accepted 12 July 2021; Published 15 July 2021

Academic Editor: Mutamed M. Ayyash

Copyright ( 2021 Belayneh Regasa Dadi et al. This is an open access article distributed under the Creative Commons Attribution License, which permits unrestricted use, distribution, and reproduction in any medium, provided the original work is properly cited.

\begin{abstract}
Background. Antimicrobials used for the treatment and prevention of bacterial infections are mainly released nonmetabolized into the aquatic environment via wastewater. Sometimes, unused therapeutic drugs are released down the drains that could act as selective pressure for the development of resistance. The aim of this study was to assess the bacteriological profile of wastewater in health facilities and determine antibiotic susceptibility patterns of bacterial isolates. Methods. A cross-sectional study was conducted from October 1 to December 26, 2020, in health facility wastewater. A total of 128 samples were collected from health facilities for bacteriological analysis and antimicrobial susceptibility testing. Result. A total of 128 samples were processed, and 81 bacterial isolates were recovered. The most common bacterial isolates were S. aureus (16/81 (19.8\%)) followed by Klebsiella spp. (15/81 (18.5\%)), E. coli (13/81 (16\%)), P. aeruginosa (10/81 (12.3\%)), Enterobacter spp. (8/81 (9.9\%)), Citrobacter spp. (7/81 (8.6\%)), coagulase-negative Staphylococcus (5/81 (6.2\%)), Salmonella spp. (5/81 (6.2\%)), and Shigella spp. (2/81 (2.5\%)). A majority of isolates were resistant to ampicillin $(62 / 81(76.5 \%))$. Only few isolates were resistant to ciprofloxacin $(11 / 81(13.6 \%))$, chloramphenicol (13/81 (16\%)), and kanamycin (8/54 (14.8\%)). A majority of bacterial isolates (57/81 (70.4\%)) were multidrug resistant (MDR). Conclusion. Wastewater from the health facilities contains antibiotic-resistant including multidrug-resistant bacteria. Therefore, health facility wastewater should be treated by appropriate wastewater treatment before being released into the environment.
\end{abstract}

\section{Background}

Medical waste is categorized into pathological waste-body fluids from surgery, infectious waste from laboratories, pharmaceutical waste-out-of-date pharmaceutical products, and chemical wastes-used solvents, disinfectants, pesticides, and diagnostic chemicals, aerosol containers and gas, and open sources used in in vitro diagnosis or nuclear medical therapy [1]. Sewage from hospitals, usually referred to as hospital waste, is defined as a special category of waste which comprises all wastes, biological or nonbiological, that are discarded from hospitals/healthcare centers and not intended for further use [2]. The important usage of water in hospitals gives significant volumes of waste loaded with microorganisms (the majority of which being pathogenic), heavy metals, toxic chemicals, and radioactive elements [3].
The amount of antibiotics present in hospitals discharge into effluent results, a selection pressure on bacteria [4]. In addition to pharmaceuticals, another type of chemical compound that is heavily used in hospitals and has consequently raised concern about potential environmental toxicity is disinfectants [5]. The discharge of resistant bacteria to the receiving environment can pose public health impacts through carrying the transmissible gene and by acting as a vector or reservoir of the resistant gene [6].

The impact of polluted sewage on the surface and groundwater is widely regarded as a serious threat to human health and environment in many developing countries of the world [7]. In Ethiopia, rapid urbanization and industrialization without deliberating environmental planning often lead to the release of industrial and hospital sewage effluent 
directly into the environment which is a major problem $[8,9]$. The aim of this study was to assess the bacteriological profile of wastewater in health facilities and determine antibiotic susceptibility patterns of bacterial isolates in Arba Minch, Ethiopia, where wastewater from the health facilities is released into the environment without treatment.

\section{Methods}

2.1. Sample Collection. A facility-based cross-sectional study was conducted from October to December 2021 at health facilities in Arba Minch, Ethiopia, namely, Arba Minch Hospital, Sikella Health Center, and Secha Health Center.

Each sample was collected four times a day from all sites in $250 \mathrm{~mL}$ cleaned and sterile microbiological glass bottles containing $0.2 \mathrm{~mL}$ of $3 \% \mathrm{w} / \mathrm{v}$ sodium thiosulphate [10]. The samples were transported within two hours in an ice box to the Microbiology and Parasitology Laboratory of Arba Minch University, College of Medicine and Health Sciences, for analysis.

2.2. Sample Size Determination and Sampling Technique. Wastewater samples were collected from the septic tank before being released to the open field according to guidelines of wastewater sampling technique [10]. A total of 128 samples were processed for bacteriological analysis and antimicrobial susceptibility testing.

2.3. Microbiological Investigations. The sample was thoroughly shaken to get a homogeneous mixture before a portion was taken for culture. Serial 10 -fold dilutions of wastewater samples were prepared in $0.85 \% \mathrm{NaCl}$ (normal saline). Serial dilution was done in order to get pure colonies. The bacteria were cultivated by plating $0.5 \mathrm{ml}$ of each of the desired serial dilutions of the bacterial suspensions, $7^{\text {th }}$ and $8^{\text {th }}\left(10^{-7}\right.$ and $\left.10^{-8}\right)$ dilutions of health facility wastewater. Duplicate samples were plated onto MacConkey (MAC) agar, Mannitol Salt Agar (MSA), Pseudomonas agar, and Selenite $\mathrm{F}$ Broth and then incubated at $37^{\circ} \mathrm{C}$ for $24-48$ hours. After incubation, based on colony morphology, representative colonies were picked and subcultured on different selective and differential media such as blood agar (BA), MacConkey (MAC) agar, Mannitol Salt agar (MSA), Xylose Lysine Deoxycholate (XLD) agar, and Pseudomonas agar. After obtaining pure colonies and recording important features, isolated organisms were further identified at the species level by biochemical tests [11].

2.4. Antibiotic Susceptibility Testing. The standard Kirby-Bauer disk diffusion method was used to determine the antimicrobial susceptibility profiles of the isolates [12]. Bacterial inocula were prepared by suspending the freshly grown bacteria in 4-5 $\mathrm{ml}$ normal saline, and the turbidity was adjusted to $0.5 \mathrm{McF}$ arland standards. Finally, the suspension was streaked onto the entire surface of the
Mueller-Hinton agar using a cotton swab to produce confluent growth. Antibiotic discs impregnated with specific amounts of commonly prescribed antimicrobial agents for patient use were then placed on the surface of the medium and aerobically incubated at $37^{\circ} \mathrm{C}$ for $18-24$ hours. The antimicrobial agents used were ceftriaxone $(30 \mu \mathrm{g})$, ciprofloxacin $(5 \mu \mathrm{g})$, ampicillin $(10 \mu \mathrm{g})$, amoxicillin $(25 \mu \mathrm{g})$, doxycycline $(30 \mu \mathrm{g})$, gentamycin $(10 \mu \mathrm{g})$, erythromycin $(15 \mu \mathrm{g})$, tetracycline $(30 \mu \mathrm{g})$, chloramphenicol $(30 \mu \mathrm{g})$, kanamycin $(30 \mu \mathrm{g})$, and ceftazidime $(30 \mu \mathrm{g})$. The zones of inhibition were measured, and interpretation was made using the National Committee for Clinical and Laboratory Standards Institute guidelines [13].

2.5. Data Quality Assurance. Reagents, culture media, and antimicrobial disks were checked for expiry date, damage, and storage problems. Culture media preparation was made based on the respective manufacturer's instructions. Five percent of media per batch was incubated at $37^{\circ} \mathrm{C}$ overnight and observed for bacterial growth. Those media which showed growth were discarded. Control strains Pseudomonas aeruginosa (ATCC 27853), Escherichia coli (ATCC 25922), and Staphylococcus aureus (ATCC 25923) were used.

2.6. Statistical Analysis. Data were entered, cleaned, and analyzed by using Statistical Package for Social Sciences (SPSS) software version 20.0. One-way ANOVAs, independent Students' $T$-test, and paired T-test were used to compare means of some parameters. A critical value of 0.05 was used for the inferential statistics.

\section{Results}

3.1. Bacteriological Analysis of Wastewater. A total of 128 samples were processed, and 81 bacterial isolates were recovered. Most bacterial isolates (66/81 (81.5\%)) were from Arba Minch Hospital followed by 9/81 (11.1\%) bacterial isolates from Sikella Health Center and 6/81 (7.5\%) bacterial isolates from Secha Health Center. The most common bacterial isolate was S. aureus (16/81 (19.8\%)) (Table 1).

3.2. Antimicrobial Resistance Patterns of Bacterial Isolates. A majority of isolates were resistant to ampicillin (62/81 $(76.5 \%))$. Only few isolates were resistant to ciprofloxacin $(11 / 81(13.6 \%))$, chloramphenicol (13/81 (16\%)), and kanamycin (8/54 (14.8\%)).

A majority of $S$. aureus isolates showed resistance to ampicillin $(11 / 16(68.8 \%))$ and tetracycline $(8 / 16(50 \%))$, but only few isolates were resistant to ciprofloxacin $(3 / 16$ $(18.8 \%))$, chloramphenicol $(2 / 16(12.5 \%))$, ceftazidime $(3 / 16$ $(18.8 \%))$, and erythromycin $(4 / 16(25 \%))$. A majority of Klebsiella species showed resistance to ampicillin (13/15 $(86.7 \%))$, but only few isolates were resistant to chloramphenicol $(2 / 15(13.3 \%))$, ciprofloxacin $(3 / 15(20 \%))$, gentamycin (3/15 (20\%)), and kanamycin (3/15(20\%)). A 
TABLE 1: Frequency of bacterial isolates from influent and effluent wastewater released from health facilities.

\begin{tabular}{|c|c|c|c|c|c|}
\hline \multirow[b]{2}{*}{ Bacterial isolates } & \multicolumn{2}{|c|}{ Arba Minch Hospital } & \multirow[b]{2}{*}{$\begin{array}{l}\text { Sikella Health Center } \\
\text { effluent frequency }(\%)\end{array}$} & \multirow[b]{2}{*}{$\begin{array}{l}\text { Secha Health Center } \\
\text { effluent frequency }(\%)\end{array}$} & \multirow[b]{2}{*}{$\begin{array}{c}\text { Total frequency } \\
(\%)\end{array}$} \\
\hline & $\begin{array}{c}\text { Influent } \\
\text { frequency }(\%)\end{array}$ & $\begin{array}{c}\text { Effluent } \\
\text { frequency }(\%)\end{array}$ & & & \\
\hline S. aureus & $7(17.5)$ & $4(15.4)$ & $3(33.3)$ & $2(33.3)$ & $16(19.8)$ \\
\hline Klebsiella spp. & $6(15)$ & $5(19.2)$ & $2(22.2)$ & $2(33.3)$ & $15(18.5)$ \\
\hline E. coli & $5(12.5)$ & $4(15.4)$ & $2(22.2)$ & $2(33.3)$ & $13(16)$ \\
\hline$P$. aeruginosa & $5(12.5)$ & $4(15.4)$ & $1(11.1)$ & - & $10(12.3)$ \\
\hline Enterobacter spp. & $5(12.5)$ & $2(7.7)$ & $1(11.1)$ & - & $8(9.9)$ \\
\hline Citrobacter spp. & $4(10)$ & $3(11.5)$ & - & - & $7(8.6)$ \\
\hline $\begin{array}{l}\text { Coagulase-negative } \\
\text { Staphylococci }\end{array}$ & $3(7.5)$ & $2(7.7)$ & - & - & $5(6.2)$ \\
\hline Salmonella spp. & $3(7.5)$ & $2(7.7)$ & - & - & $5(6.2)$ \\
\hline Shigella spp. & $2(5)$ & - & - & - & $2(2.5)$ \\
\hline Total & $40(100)$ & $26(100)$ & $9(100)$ & $6(100)$ & $81(100)$ \\
\hline
\end{tabular}

Table 2: Antibiotic susceptibility patterns of bacterial isolates from influent wastewater released from Arba Minch Hospital.

\begin{tabular}{|c|c|c|c|c|c|c|c|c|c|c|c|c|}
\hline \multirow{2}{*}{ Bacterial isolates } & \multicolumn{12}{|c|}{ Antimicrobial agents (frequency (percentage)) } \\
\hline & & AMC & AMP & $\mathrm{CPR}$ & CTR & $\mathrm{CHL}$ & CTX & GN & TET & KAN & DOX & ERY \\
\hline \multirow{3}{*}{ P. aeruginosa [5] } & $\mathrm{S}$ & - & $0(0)$ & $2(40)$ & $0(0)$ & $2(40)$ & $2(40)$ & $3(60)$ & - & $3(60)$ & - & - \\
\hline & I & - & $1(20)$ & $2(40)$ & $3(60)$ & $1(20)$ & $1(20)$ & $1(20)$ & - & $0(0)$ & - & - \\
\hline & $\mathrm{R}$ & - & $4(80)$ & $1(20)$ & $2(40)$ & $2(40)$ & $2(40)$ & $1(20)$ & - & $2(40)$ & - & - \\
\hline \multirow{3}{*}{ Klebsiella spp. [6] } & $S$ & $3(50)$ & $0(0)$ & $2(33)$ & $3(50)$ & $3(50)$ & $2(33)$ & $3(50)$ & $1(17)$ & $2(33)$ & $1(17)$ & - \\
\hline & I & $0(0)$ & $0(0)$ & $2(33)$ & $2(33)$ & $3(50)$ & $2(33)$ & $2(33)$ & $2(33)$ & $2(33)$ & $2(33)$ & - \\
\hline & $\mathrm{R}$ & $3(50)$ & $6(100)$ & $2(33)$ & $1(17)$ & $0(0)$ & $2(33)$ & $1(17)$ & $3(50)$ & $2(33)$ & $3(50)$ & - \\
\hline \multirow{3}{*}{ E. coli [5] } & $S$ & $3(60)$ & $1(20)$ & $3(60)$ & $2(40)$ & $3(60)$ & $2(40)$ & $2(40)$ & $2(40)$ & $4(80)$ & $1(20)$ & - \\
\hline & I & $0(0)$ & $0(0)$ & $2(40)$ & $1(20)$ & $1(20)$ & $1(20)$ & $2(40)$ & $1(20)$ & $1(20)$ & $2(40)$ & - \\
\hline & $\mathrm{R}$ & $2(40)$ & $4(80)$ & $0(0)$ & $2(40$ & $1(20)$ & $2(40)$ & $1(20)$ & $2(40)$ & $0(0)$ & $2(40)$ & - \\
\hline \multirow{3}{*}{ Citrobacter spp. [4] } & $\mathrm{S}$ & $3(75)$ & $0(0)$ & $2(50)$ & $2(50)$ & $2(50)$ & $2(50)$ & $2(50)$ & $2(50)$ & $2(50)$ & $2(50)$ & - \\
\hline & I & $0(0)$ & $1(25)$ & $2(50)$ & $1(25)$ & $2(50)$ & $0(0)$ & $0(0)$ & $0(0)$ & $2(50)$ & $1(25)$ & - \\
\hline & $\mathrm{R}$ & $1(25)$ & $3(75)$ & $0(0)$ & $1(25)$ & $0(0)$ & $2(50)$ & $2(50)$ & $2(50)$ & $0(0)$ & $1(25)$ & - \\
\hline \multirow{3}{*}{ Enterobacter spp. [5] } & S & $2(40)$ & $1(20)$ & $2(40)$ & $2(40)$ & $4(80)$ & $2(40)$ & $2(40)$ & $2(40)$ & $3(60)$ & $2(40)$ & - \\
\hline & I & $1(20)$ & $1(20)$ & $3(60)$ & $1(20)$ & $1(20)$ & $1(20)$ & $2(40)$ & $1(20)$ & $2(40)$ & $1(20)$ & - \\
\hline & $\mathrm{R}$ & $2(40)$ & $3(60)$ & $0(0)$ & $2(40)$ & $0(0)$ & $2(40)$ & $1(20)$ & $2(40)$ & $0(0)$ & $2(40)$ & - \\
\hline \multirow{3}{*}{ S. aureus [7] } & $\mathrm{S}$ & $2(29)$ & $1(20)$ & $3(43)$ & $4(57)$ & $6(72)$ & $3(43)$ & $4(57)$ & $0(0)$ & - & - & $3(43)$ \\
\hline & I & $1(14)$ & $0(0)$ & $2(29)$ & $0(0)$ & $1(14)$ & $2(29)$ & $0(0)$ & $4(57)$ & - & - & $1(14)$ \\
\hline & $\mathrm{R}$ & $4(57)$ & $6(80)$ & $2(29)$ & $3(43)$ & $1(14)$ & $2(29)$ & $3(43)$ & $3(43)$ & - & - & $3(43)$ \\
\hline \multirow{3}{*}{ Coagulase-negative Staphylococcus [3] } & $\mathrm{S}$ & $1(33)$ & $0(0)$ & $2(67)$ & $2(67)$ & $2(67)$ & $1(33)$ & $2(67)$ & $0(0)$ & - & - & $1(33)$ \\
\hline & I & $0(0)$ & $0(0)$ & $1(33)$ & $1(33)$ & $1(33)$ & $1(33)$ & $1(33)$ & $2(67)$ & - & - & $0(0)$ \\
\hline & $\mathrm{R}$ & $2(67)$ & $3(100)$ & $0(0)$ & $0(0)$ & $0(0)$ & $1(33)$ & $0(0)$ & $1(33)$ & - & - & $2(67)$ \\
\hline \multirow{3}{*}{ Salmonella spp. [3] } & $\mathrm{S}$ & $3(100)$ & $0(0)$ & $2(67)$ & $1(33)$ & $2(67)$ & $1(33)$ & $2(67)$ & $0(0)$ & - & $0(0)$ & - \\
\hline & I & $0(0)$ & $1(33)$ & $1(33)$ & $1(33)$ & $0(0)$ & $1(33)$ & $1(33)$ & $1(33)$ & - & $1(33)$ & - \\
\hline & $\mathrm{R}$ & $0(0)$ & $2(67)$ & $0(0)$ & $1(33)$ & $1(33)$ & $1(33)$ & $0(0)$ & $2(67)$ & - & $2(67)$ & - \\
\hline
\end{tabular}

AMC: amoxicillin; AMP: ampicillin; CPR: ciprofloxacin; CTR: ceftriaxone; CHL: chloramphenicol; CTX: ceftazidime; GN: gentamycin; TET: tetracycline; KAN: kanamycin; DOX: doxycycline; ERY: erythromycin; S: susceptible; I: intermediate; R: resistant.

majority of $E$. coli isolates showed resistance to ampicillin $(10 / 13(76.9 \%))$, but only few isolates were resistant to gentamycin $(1 / 13(7.7 \%))$, kanamycin $(1 / 13(7.7 \%))$, and ciprofloxacin $(2 / 13(15.4 \%))$. A majority of Pseudomonas species showed resistance for ampicillin (8/10 (80\%)), but only few isolates were resistant to ciprofloxacin $(2 / 10(20 \%))$, ceftriaxone $(2 / 10(20 \%))$, and gentamycin $(2 / 10(20 \%))$ (see Tables 2-5).

A majority of bacterial isolates from Arba Minch Hospital influent wastewater were resistant to ampicillin $(33 / 40(82.5 \%))$ and tetracycline $(16 / 35$ (46\%)) (see Table 2).
Among the isolated bacteria from Arba Minch Hospital effluent wastewater, Salmonella species were $100 \%$ resistant to ampicillin, coagulase-negative Staphylococci were found to be $100 \%$ resistant to ceftriaxone, and Salmonella species were $50 \%$ resistant to ceftriaxone (Table 3 ).

A majority of bacterial isolates from Sikella Health Center effluent wastewater were resistant to ampicillin (5/9 $(55 \%))$. Among bacteria isolates from Sikella Health Center effluent wastewater, $P$. aeruginosa and Enterobacter species were found to be $100 \%$ resistant to ampicillin, and Klebsiella species and E. coli were 50\% resistant to ampicillin (Table 4). 
TABle 3: Antibiotic susceptibility patterns of bacterial isolates from effluent released from Arba Minch Hospital.

\begin{tabular}{|c|c|c|c|c|c|c|c|c|c|c|c|c|}
\hline \multirow{2}{*}{ Bacterial isolates } & \multicolumn{12}{|c|}{ Antimicrobial agent (frequency (percentage)) } \\
\hline & & AMC & AMP & $\mathrm{CPR}$ & CTR & $\mathrm{CHL}$ & CTX & GN & TET & KAN & DOX & ERY \\
\hline \multirow{3}{*}{ P. aeruginosa [4] } & $\mathrm{S}$ & - & $0(0)$ & $3(75)$ & $2(50)$ & $2(50)$ & $0(0)$ & $3(75)$ & - & $3(75)$ & - & - \\
\hline & $\mathrm{I}$ & - & $1(25)$ & $0(0)$ & $2(50)$ & $1(25)$ & $2(50)$ & $0(0)$ & - & $0(0)$ & - & - \\
\hline & $\mathrm{R}$ & - & $3(75)$ & $1(25)$ & $0(0)$ & $1(25)$ & $2(50)$ & $1(25)$ & - & $1(25)$ & - & - \\
\hline \multirow{3}{*}{ Klebsiella spp. [5] } & $\mathrm{S}$ & $2(40)$ & $1(20)$ & $3(75)$ & $2(40)$ & $3(60)$ & $1(20)$ & $3(60)$ & $2(40)$ & $3(60)$ & $1(20)$ & - \\
\hline & $\mathrm{I}$ & $1(20)$ & $0(0)$ & $1(20)$ & $0(0)$ & $0(0)$ & $4(80)$ & $0(0)$ & $1(20)$ & $1(20)$ & $2(40)$ & - \\
\hline & $\mathrm{R}$ & $2(40)$ & $4(80)$ & $1(20)$ & $3(60)$ & $2(40)$ & $0(0)$ & $2(40)$ & $2(40)$ & $1(20)$ & $2(40)$ & - \\
\hline \multirow{3}{*}{ E. coli $[4]$} & $\mathrm{S}$ & $2(50)$ & $0(0)$ & $2(50)$ & $3(75)$ & $3(75)$ & $2(50)$ & $1(25)$ & $2(50)$ & $2(50)$ & $2(50)$ & - \\
\hline & I & $1(25)$ & $1(25)$ & $1(25)$ & $1(25)$ & $0(0)$ & $0(0)$ & $3(75)$ & $1(25)$ & $1(25)$ & $0(0)$ & - \\
\hline & $\mathrm{R}$ & $1(25)$ & $3(75)$ & $1(25)$ & $0(0)$ & $1(25)$ & $2(50)$ & $0(0)$ & $1(25)$ & $1(25)$ & $2(50)$ & - \\
\hline \multirow{3}{*}{ Citrobacter spp. [3] } & $\mathrm{S}$ & $2(67)$ & $0(0)$ & $2(67)$ & $1(33)$ & $2(67)$ & $0(0)$ & $2(67)$ & $2(67)$ & $2(67)$ & $1(33)$ & - \\
\hline & $\mathrm{I}$ & $0(0)$ & $1(33)$ & $1(33)$ & $1(33)$ & $1(33)$ & $1(33)$ & $1(33)$ & $0(0)$ & $0(0)$ & $1(33)$ & - \\
\hline & $\mathrm{R}$ & $1(33)$ & $2(67)$ & $0(0)$ & $1(33)$ & $0(0)$ & $2(67)$ & $0(0)$ & $1(33)$ & $1(33)$ & $1(33)$ & - \\
\hline \multirow{3}{*}{ Enterobacter spp. [2] } & $\mathrm{S}$ & $1(50)$ & $1(50)$ & $1(50)$ & $2(100)$ & $1(50)$ & $2(100)$ & $2(100)$ & $0(0)$ & $1(50)$ & $2(100)$ & - \\
\hline & I & $0(0)$ & $0(0)$ & $0(0)$ & $0(0)$ & $1(50)$ & $0(0)$ & $0(0)$ & $1(50)$ & $1(50)$ & $0(0)$ & - \\
\hline & $\mathrm{R}$ & $1(50)$ & $1(50)$ & $1(50)$ & $0(0)$ & $0(0)$ & $0(0)$ & $0(0)$ & $1(50)$ & $0(0)$ & $0(0)$ & - \\
\hline \multirow{3}{*}{ S. aureus [4] } & $\mathrm{S}$ & $2(50)$ & $1(25)$ & $2(50)$ & $2(50)$ & $2(50)$ & $1(25)$ & $3(75)$ & $0(0)$ & - & - & $2(50)$ \\
\hline & $\mathrm{I}$ & $1(25)$ & $0(0)$ & $1(25)$ & $0(0)$ & $1(25)$ & $2(50)$ & $0(0)$ & $1(25)$ & - & - & $1(25)$ \\
\hline & $\mathrm{R}$ & $1(25)$ & $3(75)$ & $1(25)$ & $2(50)$ & $1(25)$ & $1(25)$ & $1(25)$ & $3(75)$ & - & - & $1(25)$ \\
\hline \multirow{3}{*}{ CONS [2] } & $\mathrm{S}$ & $1(50)$ & $0(0)$ & $1(50)$ & $0(0)$ & $1(50)$ & $2(100)$ & $1(50)$ & $1(50)$ & - & - & $1(50)$ \\
\hline & I & $0(0)$ & $1(50)$ & $1(50)$ & $0(0)$ & $0(0)$ & $0(0)$ & $0(0)$ & $1(50)$ & - & - & $0(0)$ \\
\hline & $\mathrm{R}$ & $1(50)$ & $1(50)$ & $0(0)$ & $2(100)$ & $1(50)$ & $0(0)$ & $1(50)$ & $0(0)$ & - & - & $1(50)$ \\
\hline \multirow{3}{*}{ Salmonella spp. [2] } & $\mathrm{S}$ & $1(50)$ & $0(0)$ & $2(100)$ & $0(0)$ & $1(50)$ & $0(0)$ & $1(50)$ & $1(50)$ & - & $0(0)$ & - \\
\hline & I & $0(0)$ & $0(0)$ & $0(0)$ & $1(50)$ & $0(0)$ & $1(50)$ & $1(50)$ & $0(0)$ & - & $1(50)$ & - \\
\hline & $\mathrm{R}$ & $1(50)$ & $2(100)$ & $0(0)$ & $1(50)$ & $1(50)$ & $1(50)$ & $0(0)$ & $1(50)$ & - & $1(50)$ & - \\
\hline
\end{tabular}

AMC: amoxicillin; AMP: ampicillin; CPR: ciprofloxacin; CTR: ceftriaxone; CHL: chloramphenicol; CTX: ceftazidime; GN: gentamycin; TET: tetracycline; KAN: kanamycin; DOX: doxycycline; ERY: erythromycin; S: susceptible; I: intermediate; R: resistant.

TABle 4: Antibiotic susceptibility patterns of bacterial isolates from effluent released from Sikella Health Center.

\begin{tabular}{|c|c|c|c|c|c|c|c|c|c|c|c|c|}
\hline \multirow{2}{*}{ Bacterial isolates } & \multicolumn{12}{|c|}{ Antimicrobial agents (frequency (percentage)) } \\
\hline & & AMC & AMP & $\mathrm{CPR}$ & CTR & CHL & CTX & GN & TET & KAN & DOX & ERY \\
\hline \multirow{3}{*}{$P$. aeruginosa [1] } & $S$ & - & $0(0)$ & $1(100)$ & $1(100)$ & $1(100)$ & $1(100)$ & $1(100)$ & - & $1(100)$ & - & - \\
\hline & I & - & $0(0)$ & $0(0)$ & $0(0)$ & $0(0)$ & $0(0)$ & $0(0)$ & - & $0(0)$ & - & - \\
\hline & $\mathrm{R}$ & - & $1(100)$ & $0(0)$ & $0(0)$ & $0(0)$ & $0(0)$ & $0(0)$ & - & $0(0)$ & - & - \\
\hline \multirow{3}{*}{ Klebsiella spp. [2] } & $S$ & $2(100)$ & $0(0)$ & $2(100)$ & $2(100)$ & $2(100)$ & $2(100)$ & $2(100)$ & $2(100)$ & $2(100)$ & $2(100)$ & - \\
\hline & I & $0(0)$ & $1(50)$ & $0(0)$ & $0(0)$ & $0(0)$ & $0(0)$ & $0(0)$ & $0(0)$ & $0(0)$ & $0(0)$ & - \\
\hline & $\mathrm{R}$ & $0(0)$ & $1(50)$ & $0(0)$ & $0(0)$ & $0(0)$ & $0(0)$ & $0(0)$ & $0(0)$ & $0(0)$ & $0(0)$ & - \\
\hline \multirow{3}{*}{ E. coli $[2]$} & $S$ & $2(100)$ & $1(50)$ & $1(50)$ & $2(100)$ & $1(50)$ & $1(50)$ & $2(100)$ & $2(100)$ & $2(100)$ & $2(100)$ & - \\
\hline & I & $0(0)$ & $0(0)$ & $0(0)$ & $0(0)$ & $0(0)$ & $1(50)$ & $0(0)$ & $0(0)$ & $0(0)$ & $0(0)$ & - \\
\hline & $\mathrm{R}$ & $0(0)$ & $1(50)$ & $1(50)$ & $0(0)$ & $1(50)$ & $0(0)$ & $0(0)$ & $0(0)$ & $0(0)$ & $0(0)$ & - \\
\hline \multirow{3}{*}{ Enterobacter spp. [1] } & $S$ & $1(100)$ & $0(0)$ & $1(100)$ & $1(100)$ & $1(100)$ & $1(100)$ & $1(100)$ & $1(100)$ & $1(100)$ & $1(100)$ & - \\
\hline & I & $0(0)$ & $0(0)$ & $0(0)$ & $0(0)$ & $0(0)$ & $0(0)$ & $0(0)$ & $0(0)$ & $0(0)$ & $0(0)$ & - \\
\hline & $\mathrm{R}$ & $0(0)$ & $1(100)$ & $0(0)$ & $0(0)$ & $0(0)$ & $0(0)$ & $0(0)$ & $0(0)$ & $0(0)$ & $0(0)$ & - \\
\hline \multirow{3}{*}{ S. aureus [3] } & $S$ & $1(33)$ & $1(33)$ & $2(66)$ & $3(100)$ & $2(67)$ & $3(100)$ & $1(33)$ & $2(67)$ & - & - & $3(100)$ \\
\hline & I & $1(33)$ & $1(33)$ & $1(33)$ & $0(0)$ & $1(33)$ & $0(0)$ & $2(67)$ & $0(0)$ & - & - & $0(0)$ \\
\hline & $\mathrm{R}$ & $1(33)$ & $1(33)$ & $0(0)$ & $0(0)$ & $0(0)$ & $0(0)$ & $0(0)$ & $1(33)$ & - & - & $0(0)$ \\
\hline
\end{tabular}

AMC: amoxicillin; AMP: ampicillin; CPR: ciprofloxacin; CTR: ceftriaxone; CHL: chloramphenicol; CTX: ceftazidime; GN: gentamycin; TET: tetracycline; KAN: kanamycin; DOX: doxycycline; ERY: erythromycin; S: susceptible; I: intermediate; R: resistant.

A majority of bacterial isolates from Secha Health Center effluent wastewater were resistant to ampicillin (5/6(83.3\%)). Among bacteria isolates from Secha Health Center effluent wastewater, Klebsiella species and E. coli were $100 \%$ resistant to ampicillin (Table 5).

A majority of bacterial isolates (57/81 (70.4\%)) were multidrug resistant (MDR).

\section{Discussion}

In our study, Staphylococcus aureus were isolated in high number among wastewater samples from the health facilities continuously released to the receiving environment. Detection of E. coli from all sites may be due to the fact that E. coli is one of the commensal organisms commonly 
TABle 5: Antibiotic susceptibility patterns of bacterial isolates from effluent released from Secha Health Center.

\begin{tabular}{|c|c|c|c|c|c|c|c|c|c|c|c|c|}
\hline \multirow{2}{*}{ Bacterial isolates } & \multicolumn{12}{|c|}{ Antimicrobial agents (frequency (percentage)) } \\
\hline & & AMC & AMP & $\mathrm{CPR}$ & CTR & CHL & CTX & GN & TET & KAN & DOX & ERY \\
\hline \multirow{3}{*}{ Klebsiella spp. [2] } & $\mathrm{S}$ & $2(100)$ & $0(0)$ & $2(100)$ & $2(100)$ & $2(100)$ & $2(100)$ & $2(100)$ & $2(100)$ & $2(100)$ & $2(100)$ & - \\
\hline & I & $0(0)$ & $0(0)$ & $0(0)$ & $0(0)$ & $0(0)$ & $0(0)$ & $0(0)$ & $0(0)$ & $0(0)$ & $0(0)$ & - \\
\hline & $\mathrm{R}$ & $0(0)$ & $2(100)$ & $0(0)$ & $0(0)$ & $0(0)$ & $0(0)$ & $0(0)$ & $0(0)$ & $0(0)$ & $0(0)$ & - \\
\hline \multirow{3}{*}{ E. coli $[2]$} & S & $2(100)$ & $0(0)$ & $1(50)$ & $2(100)$ & $2(100)$ & $2(100)$ & $2(100)$ & $2(100)$ & $2(100)$ & $1(50)$ & - \\
\hline & I & $0(0)$ & $0(0)$ & $1(50)$ & $0(0)$ & $0(0)$ & $0(0)$ & $0(0)$ & $0(0)$ & $0(0)$ & $0(0)$ & - \\
\hline & $\mathrm{R}$ & $0(0)$ & $2(100)$ & $0(0)$ & $0(0)$ & $0(0)$ & $0(0)$ & $0(0)$ & $0(0)$ & $0(0)$ & $1(50)$ & - \\
\hline \multirow{3}{*}{ S. aureus [2] } & S & $2(100)$ & $0(0)$ & $2(100)$ & $1(50)$ & $2(100)$ & $1(50)$ & $2(100)$ & $1(50)$ & - & - & $2(100)$ \\
\hline & I & $0(0)$ & $1(50)$ & $0(0)$ & $1(50)$ & $0(0)$ & $1(50)$ & $0(0)$ & $0(0)$ & - & - & $0(0)$ \\
\hline & $\mathrm{R}$ & $0(0)$ & $1(50)$ & $0(0)$ & $0(0)$ & $0(0)$ & $0(0)$ & $0(0)$ & $1(50)$ & - & - & $0(0)$ \\
\hline
\end{tabular}

AMC: amoxicillin; AMP: ampicillin; CPR: ciprofloxacin; CTR: ceftriaxone; CHL: chloramphenicol; CTX: ceftazidime; GN: gentamycin; TET: tetracycline; KAN: kanamycin; DOX: doxycycline; ERY: erythromycin; S: susceptible; I: intermediate; R: resistant.

available in the gastrointestinal tract of humans and animals. Direct and indirect fecal contamination of wastewater from hospital and health centers can easily contaminate the receiving water bodies with potential pathogenic E. coli as well as multidrug-resistant strains which can horizontally disseminate to other organisms. The same result was observed in Ethiopia as E. coli was detected in high concentration from the effluents of wastewater $[14,15]$. Another study done in India showed large numbers of enteric bacteria, and $S$. aureus and $P$. aeruginosa were recovered from wastewater [16]. Similarly, studies done in Thailand [17], Nigeria $[18,19]$, Tunisia [20], and Ethiopia [15] showed that Pseudomonas aeruginosa, E. coli, Staphylococcus aureus, and Salmonella species were the predominant bacteria isolated from health facility wastewater. This may be due to inefficient removal of pathogenic bacteria by oxidation pond or admission of a large number of cases with these bacterial infections [15].

High numbers of bacteria were isolated from health facility wastewater samples. This is an indication of poor and inefficient management of wastewater in community health facilities. The absence of health facility wastewater treatment before releasing wastewater into the sewage system may contribute to the dissemination of such multidrug-resistant bacteria from the health facilities to the environment by draining those bacteria into the city sewage pool or directly into the water bodies such as lakes and rivers. The persistence of large amounts of antibiotics in the environment poses a serious threat to the ecosystem as these could enhance resistance in microbes, which may result in an increase in disease burden along with the change in the structure of the microbial community.

In our study, a majority of bacterial isolates (70.4\%) were multidrug resistant (MDR). A similar study conducted in India showed simultaneous resistance of isolates for ampicillin, combination of ampicillin with clavulanic acid, cotrimoxazole, tetracycline, and cephalosporins first, second, and third generation in the final effluent of the wastewater treatment plant [21]. Health facility wastewater contains a diverse group of pathogenic, potentially pathogenic, and environmental bacteria. The characteristic composition makes sewage particularly a suitable environmental condition for the growth and spread of antibiotic resistance due to selection pressure and horizontal gene transfer [22-24].

The present study showed that most bacterial isolates from hospital wastewater show a higher rate of resistance than bacterial isolates from health center wastewater. However, other studies reported that a higher rate of resistance in bacterial isolates from the final effluent of the wastewater treatment plant was found $[25,26]$. A similar finding showed that waste effluent from health facilities contains high numbers of drug-resistant bacteria [27].

4.1. Limitations of the Study. Although the filter paper is important to filter the microorganisms from the liquid, it helps to easily diagnose the organisms; this study failed to filter the sample due to the lack of the pore membrane.

\section{Conclusions}

High numbers of drug-resistant including multidrug-resistant bacteria were isolated from health facility wastewater samples. Therefore, health facility wastewater should be treated by an appropriate wastewater treatment plant before being released into the environment to minimize dissemination of pathogenic and potentially pathogenic bacteria to the receiving environment.

\section{Data Availability}

The datasets generated and/or analyzed during the current study are not publicly available due to ethical and confidentiality reasons but are available from the corresponding author upon reasonable request under the ethics committee's approval.

\section{Ethical Approval}

Ethical clearance was obtained from the Institutional Research Ethics Review Board (IRB), College of Medicine and Health Sciences, Arba Minch University, and permission was obtained from concerned authorities such as Arba Minch Hospital, Sikella Health Center, and Secha Health Center administrative bodies. 


\section{Disclosure}

The funding agencies had no involvement in the design of the study, data collection and analysis, interpretation of the data, and writing of the manuscript.

\section{Conflicts of Interest}

The authors declare that they have no conflicts of interest.

\section{Authors' Contributions}

BRD, EG, MT, and MS carried out proposal development, data collection, and data analysis and drafted the manuscript. All authors read and approved the final manuscript.

\section{Acknowledgments}

The authors would like to thank those who were involved in this research. This research was supported by Arba Minch University.

\section{References}

[1] W. H. Organization, Status of Health-Care Waste Management in Selected Countries of the Western Pacific Region, Manila: WHO Regional Office for the Western Pacific, Manila, Philippines, 2015.

[2] S. Oyeleke, N. Istifanus, and S. Manga, "The effects of hospital solid waste on the receiving environment," International Journal of Integrative Biology, vol. 3, no. 3, p. 191, 2008.

[3] K. Kümmerer, "Resistance in the environment," Journal of Antimicrobial Chemotherapy, vol. 54, no. 2, pp. 311-320, 2004.

[4] H. Beyene and G. Redaie, "Assessment of waste stabilization ponds for the treatment of hospital wastewater: the case of Hawassa University Referral Hospital," World Applied Sciences Journal, vol. 15, no. 1, pp. 142-150, 2011.

[5] HHSD Bank, National Library of Medicine. Toxicology Information Program, Bank HHSD, Bethesda, MD, USA, 1996.

[6] L. Nuñez and J. Moretton, "Disinfectant-resistant bacteria in Buenos Aires city hospital wastewater," Brazilian Journal of Microbiology, vol. 38, no. 4, pp. 644-648, 2007.

[7] E. Gerlach and R. Franceys, "Regulating water services for the poor: the case of Amman," Geoforum, vol. 40, no. 3, pp. 431-441, 2009.

[8] M. L. Tadesse and A. Kumie, "Healthcare waste generation and management practice in government health centers of Addis Ababa, Ethiopia," BMC Public Health, vol. 14, no. 1, p. 1221, 2014.

[9] F. Engdaw, "Physioco-chemical parameters and bacteriological qualities of water samples from wastewater treatment pond, University of Gonder, Ethiopia," International Journal of Pharmaceutical and Health Care Research, vol. 2, pp. 192-197, 2014.

[10] A. W. P. C. F. APHA, Standard Methods for the Examination of Water and Wastewater, American Public Health Association, Washington, DC, USA, 2005.

[11] W. H. Organization, Guidelines for Antimicrobial Resistance Surveillance, WHO Regional Office for the Eastern Mediterranean, Cairo, Egypt, 1996.

[12] A. W. Bauer, W. M. Kirby, J. C. Sherris, M. turck, and M. Turck, "Antibiotic susceptibility testing by a standardized single disk method," American Journal of Clinical Pathology, vol. 45, no. 4, pp. 493-6, 1966.

[13] P. Wayne, Clinical and Laboratory Standards Institute (CLSI): Performance Standards for Antimicrobial Susceptibility Testing: Twenty-First Informational Supplement. CLSI-2012-P, , pp. 100-122, Clinical and Laboratory Standards Institute (CLSI), 2014

[14] H. A. Masaadeh and A. S. Jaran, "Determination of the antibacterial efficacy of common chemical agents in cleaning and disinfection in hospitals of north Jordan," American Journal of Applied Sciences, vol. 6, no. 5, pp. 811-815, 2009.

[15] S. Fekadu, Y. Merid, H. Beyene, W. Teshome, and S. GebreSelassie, "Assessment of antibiotic- and disinfectant-resistant bacteria in hospital wastewater, south Ethiopia: a cross-sectional study," Journal of Infection in Developing Countries, vol. 9, no. 2, pp. 149-156, 2015.

[16] V. Chitnis, D. Chitnis, S. Patil, and R. Kant, "Hospital effluent: a source of multiple drug-resistant bacteria," Current Science, vol. 79, pp. 989-991, 2000.

[17] S. Danchaivijitr, W. Wongchanapai, S. Assanasen, and D. Jintanothaitavorn, "Microbial and heavy metal contamination of treated hospital wastewater in Thailand," Journal of the Medical Association of Thailand, vol. 88, no. 10, pp. S59-S64, 2005.

[18] S. Onuoha, "Isolation and characterization of multi-drug resistant bacterial pathogens from hospital effluents, south eastern, Nigeria," World Applied Sciences Journal, vol. 35, pp. 82-87, 2017.

[19] A. A. Elmanama, A. Y. ElKichaoui, and M. M. Mohsin, "Contribution of hospital wastewater to the spread of antibiotic resistance in comparison to non-health," Journal of AlAqsa University, vol. 10, no. 1, pp. 108-121, 2006.

[20] I. B. Salem, I. Ouardani, M. Hassine, and M. Aouni, "Bacteriological and physico-chemical assessment of wastewater in different region of Tunisia: impact on human health," BMC Research Notes, vol. 4, no. 1, p. 144, 2011.

[21] M. Katouli, J. Thompson, A. Gündoğdu, and H. Stratton, Eds., Science Forum and Stakeholder Engagement: Building Linkages, Collaboration and Science Quality, Griffith University, Brisbane, Australia, 2012.

[22] D. Periasamy and A. Sundaram, "A novel approach for pathogen reduction in wastewater treatment," Journal of Environmental Health Science and Engineering, vol. 11, no. 1, p. 12, 2013.

[23] R. Cantón, J. P. Horcajada, A. Oliver, P. R. Garbajosa, and J. Vila, "Inappropriate use of antibiotics in hospitals: the complex relationship between antibiotic use and antimicrobial resistance," Enfermedades Infecciosas Y Microbiología Clínica, vol. 31, pp. 3-11, 2013.

[24] J. Davies and D. Davies, "Origins and evolution of antibiotic resistance," Microbiology and Molecular Biology Reviews, vol. 74, no. 3, pp. 417-433, 2010.

[25] P. Servais and J. Passerat, "Antimicrobial resistance of fecal bacteria in waters of the Seine river watershed (France)," Science of the Total Environment, vol. 408, no. 2, pp. 365-372, 2009.

[26] A. R. Blanch, J. L. Caplin, A. Iversen et al., "Comparison of enterococcal populations related to urban and hospital wastewater in various climatic and geographic European regions," Journal of Applied Microbiology, vol. 94, no. 6, pp. 994-1002, 2003.

[27] F. Moges, M. Endris, Y. Belyhun, and W. Worku, "Isolation and characterization of multiple drug resistance bacterial pathogens from waste water in hospital and non-hospital environments, Northwest Ethiopia," BMC Research Notes, vol. 7, no. 1, p. 215, 2014. 